\title{
THE RELIABILITY OF MOBILE MULTIMEDIA MESSAGING (MMS) FOR DECISION MAKING IN DISTAL RADIUS FRACTURES: AN EFFECTIVE ALTERNATIVE
}

\author{
Dr Gayan Padmasekara MBBS ${ }^{1}$, Dr Navid Nazarian MBBS ${ }^{1}$, Dr Christopher Wall MBBS ${ }^{1}$ \\ ${ }^{1}$ Department of Orthopaedics, Southern Health, Melbourne, Australia \\ Corresponding Author: gayan@padmasekara.com
}

Background: Assessment of radiology is an integral part of fracture management in orthopaedic surgery. Mobile multimedia messaging (MMS) can be utilised in the referral process of patients with fractures. However, the reliability of using MMS in making management decisions in distal radius fractures has not been assessed.

Methods: Radiographs of 20 consecutive cases of distal radius fractures managed with closed reduction and plaster, and 20 cases managed with open reduction and internal fixation (ORIF) were analysed retrospectively by two blinded orthopaedic surgeons on a computer and anApple iPhone $3 \mathrm{GS}$. The interand intra-rater agreements were assessed.

Results: Inter-rater agreement between the MMS group and the picture archiving and communication system (PACS) group were consistent among the surgeons $(\mathrm{K}-0.55,0.80, \mathrm{p}<0.001)$. However, the management decisions were different in seven cases $(8.75 \%)$ in the study group.

Conclusion: MMS x-ray pictures can be useful in formulating management plans for patients with distal radius fractures. With advancing technology smartphones will likely play a larger role in the communication process in delivering orthopaedic care.

\section{Introduction}

Smartphones are rapidly becoming an integral part of modern society, revolutionising the portability of information with functionalities such as high quality phone cameras, internet access and access to third party services. They also have the potential to positively impact healthcare with easier access to telemedicine. ${ }^{1}$

Fracture management in orthopaedics relies on accurate diagnosis with relevant history, clinical examination, and evaluating imaging modalities such as X-rays. In busy trauma units quick ascertainment of accurate information is vital for care. Relevant history taking, and to some extent clinical examination can be directed when receiving a referral from an emergency department. However, adequate assessment of radiographs via a verbal description can be highly variable and inaccurate. This is dependent on the referrer, with variability based on experience, knowledge, language skills and the quality of the images. ${ }^{2}$ Picture archiving and communication systems (PACS) have revolutionised fracture care by providing easy access to orthopaedic surgeons and registrars to imaging findings. However, access to a PACS system may not always be available especially 
Fra JMTM

when receiving referrals at night or from other health networks.

In these instances multimedia messaging service (MMS) can be utilized to transmit a picture of the radiological images to the specialist staff. ${ }^{3,4}$ Smartphones with cameras are readily available among doctors5, and especially among junior doctors who are usually in the forefront of an emergency department. It is relatively easy to take a picture of a radiological image on a screen or film with a smartphone and to send it via MMS to the specialist staff.

Studies have evaluated the use of MMS in different aspects of surgery including plastics, neurosurgery and orthopaedics. ${ }^{6,7,9}$ A recent study evaluated the reliability of MMS in classifying wrist fractures, and foundsimilar agreement rates between observers viewing mobile phone images and x-rays. ${ }^{10}$ However, classification systems of wrist fractures are cumbersome and are rarely used in clinical practice. Furthermore, there is poor inter and intra-observer reliability among distal radius classification systems. ${ }^{11,12}$ A more relevant clinical outcome is whether the management following reviewing an image via MMS is reliable when compared to viewing the images via a PACS system. To that end, a retrospective study was designed to assess whether MMS was reliable in predicting the recommended management when compared to PACS.

\section{Methods}

Forty consecutive distal radius fractures were identified between October 2010 and January 2011. The study inclusion criteria were patients between the ages of 20 and 70 years who sustained distal radius fractures that had presented to the emergency department. Twenty consecutive cases that were managed conservatively with closed reduction and plaster, and twenty consecutive cases that required open reduction and internal fixation were selected. Pre-operative $\mathrm{x}$-rays were obtained through the hospital PACS network. Through a random number generator, three random sequences of the forty cases were created.

Two of the random sequences were used for x-ray images for the computer component of the study, where the blinded pre-operative antero-posterior (AP) and lateral images of each fracture were included in a computer presentation. The computer component was the control group, where the x-ray images of the forty cases were viewed on a standard computer monitor. The third sequence was used for the study group, which involved obtaining digital photographs of the
AP and lateral images of each fracture via a smartphone camera. The study utilized the iPhone 3GS (Apple Inc, California), which is popular with doctors in Australia and is readily available. The iPhone 3GS has a 3.2mega-pixel camera with autofocus capabilities and the images were transmitted in medium quality format via MMS on a $3 \mathrm{G}$ network. Analysing the $\mathrm{x}$-ray images on the iPhone $3 \mathrm{GS}$ by viewing the MMS pictures (screen resolution $480 \times 320,163$ ppi) comprised the study group.

The study recruited two consultant orthopaedic surgeons, who are routinely involved in trauma orthopaedics with similar levels of experience at our institution. Both surgeons were not part of the orthopaedic service during the period when the forty patients were selected, and hence were not involved in their care or decision making process at presentation. Each surgeon was provided with a universal serial bus (USB) flash-drive with the two computer presentations and an Apple ${ }^{\circledR}$ iPhone 3GS with the MMS images available in the random sequence. Both surgeons were blinded to the management that the patients had received originally.

Each surgeon was requested to complete a questionnaire where they were asked to select whether the patients should undergo closed reduction and plaster, or open reduction and internal fixation (ORIF) for their fractures. The decision was asked to be purely based on the imaging provided, however, an assumption was made that each fracture involved the non-dominant hand in an otherwise healthy patient. All injuries were assumed to have been closed and the patients were neurovascularly intact. If a decision was unable to be made from the images available they were allowed to select 'unsure' as an option. The surgeons completed the questionnaires for each part of the study on different days.

The data was collected and was analysed through the computer statistical software, MedCalc ${ }^{\circledR}$ (Version 12, MedCalc Software, Belgium). Inter-rater and intrarater agreement was calculated using kappa $(\mathrm{K})$ values, and average measures interclass correlation coefficients (ICC) were used to assess intra-rater reliability. A post hoc power analysis was performed to analyse the adequacy of the sample size.

The $\mathrm{K}$ values were interpreted as per table $1 .^{13}$ 
fi) MTM

\begin{tabular}{cc}
\hline Value of $\boldsymbol{K}$ & Strength of Agreement \\
\hline$<0.20$ & Fair \\
$0.21-0.40$ & Moderate \\
$0.41-0.60$ & Good \\
$0.61-0.80$ & \\
$0.81-1.00$ & Very Good \\
\hline
\end{tabular}

Table 1 : Interpretation of $K$ values

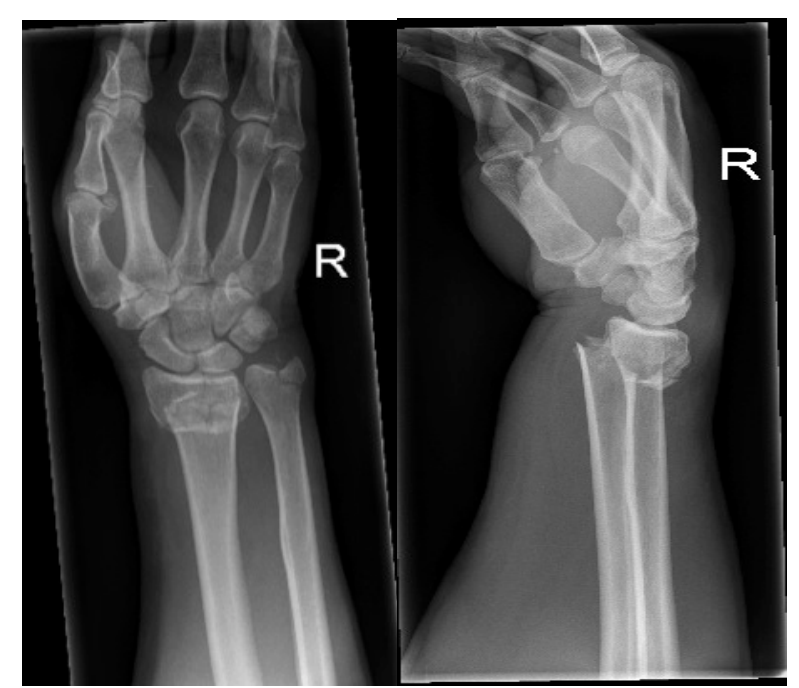

Figure 1: AP and Lateral views of distal radius fractures obtained from PACS

\section{ORIGINAL ARTICLE}

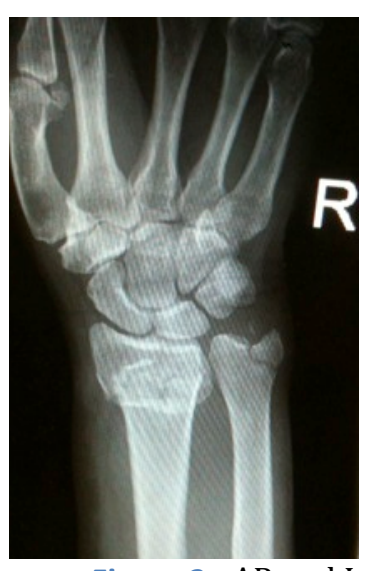

Figure 2: AP and Lateral views of distal radius fractures via MMS

\section{Results}

The twenty patients managed conservatively (mean age: 51 years (SD 14.5), range: 21-69) were comparable with the twenty patients managed operatively (mean age: 46 years (SD 14.7), range: 25 69). The inter- and intra-rater agreement values are shown in Tables 2 and 3.

\begin{tabular}{lcc}
\hline & Surgeon A & Surgeon B \\
& $(K$-value $)$ & $(K$-value $)$ \\
\hline Computer 1 vs Computer $\mathbf{2}$ & 0.899 & 0.914 \\
Computer 1 vs MMS & 0.795 & 0.552 \\
Computer 2 vs MMS & 0.798 & 0.552 \\
\hline
\end{tabular}

\section{Table 2 : Inter-rater agreement}

Inter-rater agreement was excellent within the control groups, where the images were assessed on a standard computer screen via the PACS images by both surgeons $(\mathrm{K}-0.90,0.91 ; \mathrm{p}<0.0001)$. The inter-rater agreement between the study group and the control groups were consistent among the surgeons (K- 0.55 , 0.80 respectively, $\mathrm{p}<0.001)$. However, the intra-rater agreement was varied in the study group between the two surgeons ( $\mathrm{K}-0.69, \mathrm{p}<0.0001)$, whereas the control groups had very good intra-rater agreement (K- 0.91, 0.82, $\mathrm{p}<0.0001$ ). 


\begin{tabular}{ccccc}
\hline & & \multicolumn{3}{c}{ Surgeon B } \\
& & Computer 1 & Computer 2 & MMS \\
\hline \multirow{2}{*}{ Surgeon A } & Computer 1 & 0.91 & - & - \\
$(K$-value $)$ & Computer 2 & - & 0.82 & - \\
& MMS & - & - & 0.69 \\
\hline
\end{tabular}

Table 3 : Intra-rater agreement

Between the study and control groups, MMS led to different management decisions in seven patients $(8.8 \%, \mathrm{n}=80)$. Three $(3.75 \%)$ patients were assigned to conservative management, where reviewing the PACS images deemed that they required surgery, and four $(5 \%)$ patients were opted for surgery instead of conservative management. Three $(3.8 \%)$ cases were selected as being 'unsure' in the MMS group compared to two $(2.5 \%)$ in the computer group. Only surgeon B had selected the 'unsure' option, and when these five patients were excluded in a sensitivity analysis, the inter-observer reliability improved to $\mathrm{K}$ of 1.00 for PACS images, and 0.72 for MMS. Interestingly,when comparing the management that the patients had received and the study recommendations, both the MMS and the computer groups were similar (ICC0.83 vs. 0.88 respectively, $\mathrm{p}<0.0001)$.

A post hoc power analysis was performed with the worse-case scenario results: correlation of 0.55 , alpha value of 0.05 and beta of 0.20 . A sample size of 23 would have been sufficient to reach significance.

\section{Discussion}

Distal radius fractures are one of the commonest fractures faced by orthopaedic surgeons. Careful attention to detail when reviewing radiology is perhaps more important in making management decisions for these fractures compared to other common limb fractures. Surgery is commonly recommended in the presence of radiological findings such as shortening, angulation and articular defects. As such, distal radius fractures were used as the focus of this study. The number of cases where a management decision was unable to be made was similar between the study and control groups $(n=3$ versus 2 respectively), which suggests that the clarity of MMS images is sufficient.
The use of smartphones is spreading, especially among the new generation of doctors. Eighty-five per cent of ACGME trainees in the United States owned a smartphone, where the iPhone was the most popular at $56 \%{ }^{5}$ The study utilized the iPhone 3GS, however, the newer version, iPhone 4GS has better camera capabilities and superior screen resolution. Other smartphones in the market also encompass excellent camera functionality and screen resolutions. It would be an interesting focus of research to assess whether improved technology leads to more consistent decision making or not. Initial limitations to telemedicine, especially the cost of setup are being dissolved by the widespread availability of these devices, their ease of use and advanced functionality. Smartphones will likely change the availability and access to specialist healthcare especially in rural and remote communities. ${ }^{1}$

The quality of the radiology is dependent on the quality of the images obtained. In orthopaedics, plasters can routinely affect the picture quality. The current study did not evaluate the quality of the initial images and whether this had an impact on the photographs taken by the camera phone. It is unclear whether taking a digital photograph of an already poor quality image will further reduce its ability to be interpreted. Future research is required to address this issue.

The variability between the initial treatment received by the patients and the current treatment recommendation in the study may be due to surgeon preference. The surgeons involved in the study were younger consultants, who may perhaps have a stronger inclination towards surgical management of distal radius fractures. Furthermore, patient factors are an important determinant of management decisions that were not considered in the study.

The major strength of the current study is thatthe endpoint was assessing management decisions, rather than accuracy of fracture classification. This is a more clinically relevant endpoint. It's retrospective nature (although neither surgeon was involved in the initial management of the patients) and recall bias are potential weaknesses. Other common fractures such as ankle fractures or neck of femur fractures should also be the focus of future research to assess the extent to which the findings can be generalised.

\section{Conclusion}

MMS with smartphones is a useful tool in assessing radiology images to formulate management plans in distal radius fractures. It can positively impact the communication of information from emergency 
F. JMTM

department staff to orthopaedic surgeons to make effective treatment decisions

\section{References}

1. Lambrecht CJ, Canham WD, Gattey PH, McKenzie GM. Telemedicine and orthopaedic care. A review of 2 years of experience. Clin Orthop Relat Res 1998(348):228-32.

2. McLauchlan CA, Jones K, Guly HR. Interpretation of trauma radiographs by junior doctors in accident and emergency departments: a cause for concern? J Accid Emerg Med 1997;14(5):295-8.

3. Tangtrakulwanich B, Kwunpiroj W, Chongsuvivatwong V, Geater AF, Kiatsiriroj N. Teleconsultation with digital camera images is useful for fracture care. Clin Orthop Relat Res 2006;449:308-12.

4. Eranki V, Munt J, Lim MJ, Atkinson R. Consultation of orthopaedics cases using multimedia messaging services. Open Orthop J 2010;4:164-8.

5. Franko OI, Tirrell TF. Smartphone App Use Among Medical Providers in ACGME Training Programs. $J$ Med Syst 2011.

6. Hsieh CH, Tsai HH, Yin JW, Chen CY, Yang JC, Jeng SF. Teleconsultation with the mobile camera-phone in digital soft-tissue injury: a feasibility study. Plast Reconstr Surg 2004;114(7):1776-82.

7. Waran V, Bahuri NF, Narayanan V, Ganesan D, Kadir KA. Video clip transfer of radiological images using a mobile telephone in emergency neurosurgical consultations (3G Multi-Media Messaging Service). Br J Neurosurg 2011.

8. Archbold HA, Guha AR, Shyamsundar S, McBride SJ, Charlwood P, Wray R. The use of multi-media messaging in the referral of musculoskeletal limb injuries to a tertiary trauma unit using: a 1-month evaluation. Injury 2005;36(4):560-6.

\section{ORIGINAL ARTICLE}

9. Elkaim M, Rogier A, Langlois J, Thevenin-Lemoine C, Abelin-Genevois K, Vialle R. Teleconsultation using multimedia messaging service for management plan in pediatric orthopaedics: a pilot study. $J$ Pediatr Orthop 2010;30(3):296-300.

10. Ferrero A, Garavaglia G, Gehri R, Maenza F. Analysis of the inter- and intra-observer agreement in radiographic evaluation of wrist fractures using the multimedia messaging service. Hand 2011;6:384-89.

11. Kural C, Sungur I, Kaya I, Ugras A, Erturk A, Cetinus E. Evaluation of the reliability of classification systems used for distal radius fractures. Orthopedics 2010;33(11):801.

12. Flikkila T, Nikkola-Sihto A, Kaarela O, Paakko E, Raatikainen T. Poor interobserver reliability of $\mathrm{AO}$ classification of fractures of the distal radius. Additional computed tomography is of minor value. J Bone Joint Surg Br 1998;80(4):670-2.

13. Fleiss J, Levin B, Paik M. Statistical methods for rates and proportions. 3rd ed. New York: John Wiley \& Sons, 2003.

\section{Disclaimer}

All product names, logos, brands and other trademarks featured or referred to within the Journal of Mobile Technology in Medicine e-publication remain the property of their respective trademark holders.

These trademark holders are not affiliated with the Journal of Mobile Technology in Medicine, and do not sponsor or endorse this journal. 